\title{
Cerebellar Mucormycosis in a Young Man: A Case Report
}

\section{Hossein Mashhadinezhad ${ }^{1}$ and Mohammad Ali Abouei Mehrizi ${ }^{2, *}$}

${ }^{1}$ Professor of Neurosurgery, Department of Neurosurgery, Ghaem Hospital, Mashhad University of Medical Sciences, Mashhad, Iran

${ }^{2}$ Resident of Neurosurgery, Ghaem Hospital, Faculty of Medicine, Mashhad University of Medical Sciences, Mashhad, Iran

"Corresponding author: Mohammad Ali Abouei Mehrizi, Resident of Neurosurgery, Ghaem Hospital, Faculty of Medicine, Mashhad University of Medical Sciences, Mashhad, Iran. E-mail: drabouei@yahoo.com

Received 2016 November 01; Revised 2017 February 13; Accepted 2017 March 14.

\begin{abstract}
Cerebellar mucormycosis is a rapidly progressing fungal infection that often involves immunocompromised patients. Herein, we present the case of a 20-year-old man with history of type-I diabetes mellitus and thalassemia major, who presented with headache, fever, and cough and gradually developed vertigo and ataxia during the course of admission. His imaging studies showed a spaceoccupying lesion in the posterior fossa and therefore he underwent craniotomy and the mass was removed. The pathological study revealed the mucormycosis infection of the cerebellum with small necrotic foci. The patient had no neurological signs or symptoms after the operation and he was discharged in good conditions after three weeks. He had no sign of recurrence and the MRI showed complete resolution of the lesion after follow-up.
\end{abstract}

Keywords: Mucormycosis, Cerebellar Mucormycosis, Thalassemia, Diabetes

\section{Introduction}

Mucormycosis is a rare acute fungal infection with progressive course. It mostly occurs in patients with poorly controlled diabetes mellitus, hematological malignancies or in patients receiving immunosuppressive treatment after organ transplantation and intravenous drug abusers. High levels of serum iron and treatment with deferoxamine have been also reported as predisposing factors to this infection $(1,2)$.

The primary site of infection is usually the rhinosinusal mucosa, which is contaminated following the inhalation of fungal spores. The infection can extend rapidly and infect the orbit, paranasal sinuses, and finally the brain. In some rare cases, the brain can be involved as an isolated infection, in the absence of rhinosinusal infection. It is believed that hematogenous spreading of spores in intravenous (IV) drug abusers or from pulmonary foci can lead to direct involvement of the brain $(2,3)$.

We present a patient with a history of thalassemia major, receiving treatment with deferoxamine, and type-1 diabetes mellitus, who developed slowly progressive cerebellar mucormycosis.

\section{Case Presentation}

A 20-year-old man presented with chronic headache, intermittent fever, and non-productive cough two months before his admission to the pulmonology ward of Ghaem hospital. The patient had a history of $\beta$-thalassemia major and splenectomy at the age of six years. He received blood transfusion every 3 weeks and deferoxamine capsule 1 gram daily, on a regular basis. He was diagnosed with type-1 diabetes mellitus, 7 years ago and thus received 50 IU of regular insulin daily. However, his diabetes was controlled at the time of admission and he had a hemoglobin A1C (HbA1C) of $7.5 \mathrm{mg} / \mathrm{dL}$.

The chest X-ray showed a mass located at the top of the left lung (Figure 1). A complete blood cell count revealed leukocytosis with predominance of polymorphonuclear (PMN) cells. Erythrocyte sedimentation rate (ESR) was 74 with a $2+$ positive $\mathrm{C}$-reactive protein (CRP). The antibiotic therapy was commenced and a CT-guided biopsy was performed. Further pathological studies showed inflammatory lymphocytic infiltration in the excised tissue sample. During the course of hospitalization, the patient developed vertigo and imbalance. Computerized tomography (CT)-scan and magnetic resonance imaging were performed and MRI showed a space-occupying lesion in the left hemisphere of the cerebellum (Figure 2).

The patient was admitted to the neurosurgery ward with the diagnosis of a cerebellar space-occupying lesion. Craniectomy of the posterior cranial fossa was performed. During the operation, the lesion was discovered with grey inflammatory tissue with small necrotic foci and adhesions to dura mater. The lesion had ill-defined borders, so decompression of the lesion was performed. On the third day after the operation, the report of pathological study re- 


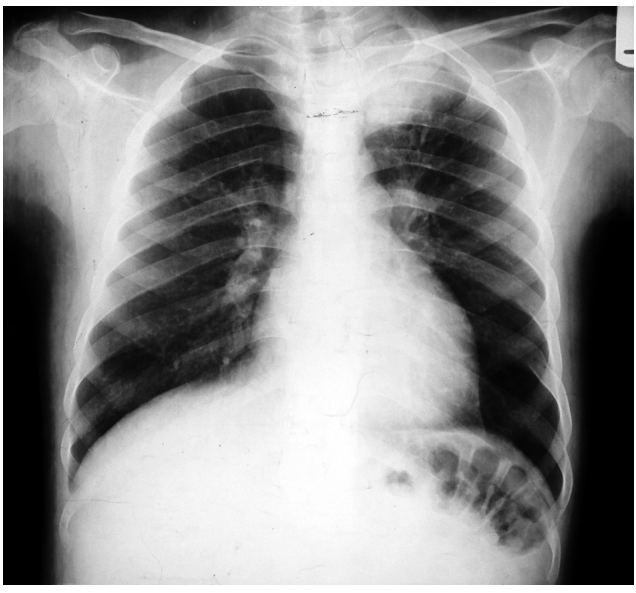

Figure 1. Patient's Pre-Operation Chest X-Ray Showing a Mass at the Top of the Left Lung

vealed extensive angioinvasion and multiple necrotic foci with irregular nonseptate hyphae of Mucormycetes (Figure 3). Moreover, the culture of the lesion was negative for bacteria but positive for Mucor species.

Based on consultation with an infectious disease specialist, the treatment was started with amphotericin deoxycolate ( $1 \mathrm{mg} / \mathrm{kg} /$ day) for three weeks and deferasirox (1000 mg/day) for two weeks. One week after initiation of deferasirox treatment, the patient's serum creatinine concentration began to rise and thus the treatment was ceased. After the treatment was over, the patient was discharged from neurosurgery ward with no neurological symptoms. His lung mass relatively decreased in size after antifungal treatment and the patient was referred to thoracic surgery ward for management of this pulmonary lesion. After a one-year follow-up, the patient's MRI showed that the brain lesion was completely resolved with no sign of recurrent infection (Figure 4).

\section{Discussion}

Mucormycosis of the brain is a rare disease caused by mucorales. The involvement of cerebellum is extremely rare and only a few cases have reported this condition so far (1). The brain is usually involved secondary to the spread of the infection from orbit and adjacent paranasal sinuses to the frontal lobe of the brain, though some cases of brain mucormycosis have been reported without rhino-orbital disease. In these cases, the brain is infected subsequent to hematogenous spread of mucorales from pulmonary foci or due to intravenous drug abuse (4-6). Air et al. have reported a case of cerebellar involvement without a primary focus and the patient had a history of intravenous drug
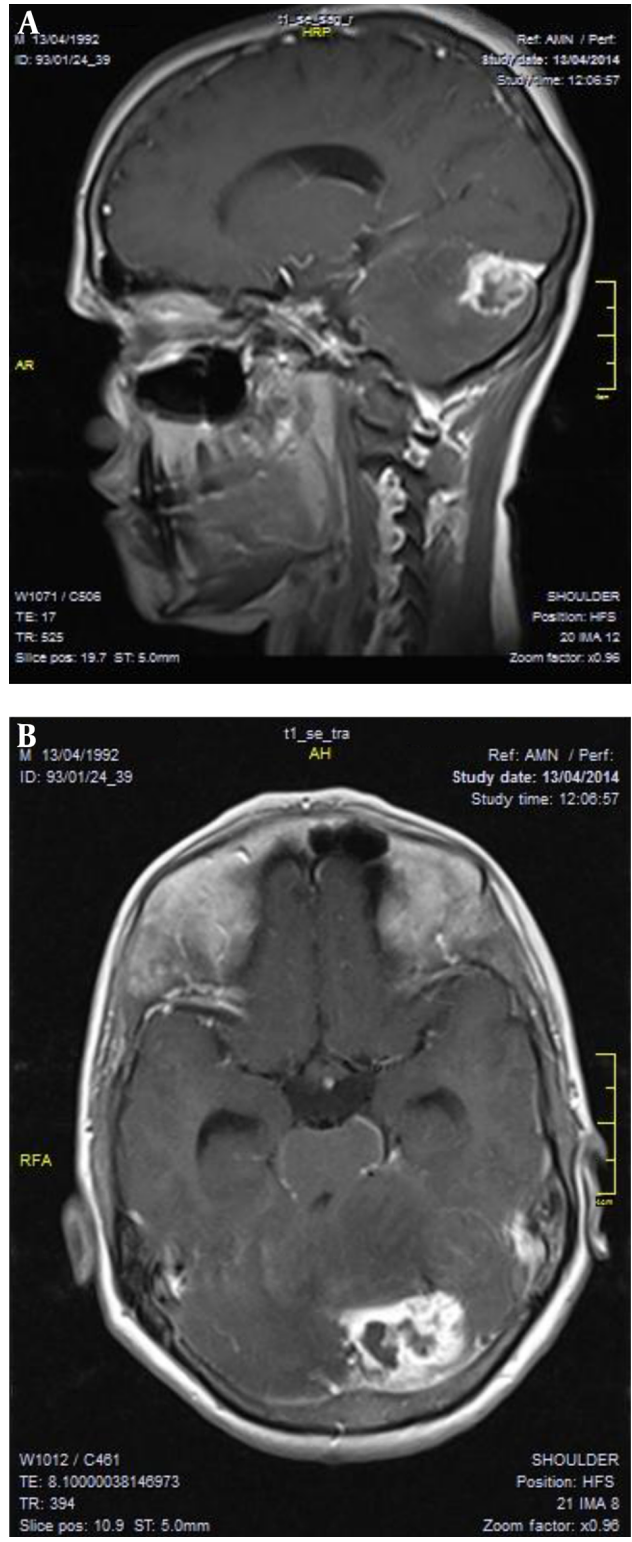

Figure 2. Sagittal (A) and Axial (B) Preoperative MRI of the Patient Showing a SpaceOccupying Lesion in the Posterior Fossa

abuse (1). Regarding the pulmonary mass and the results of the biopsy, it is highly probable that the primary source of infection in our patient is pulmonary and it could be disseminated through hematogenous spread.

The involvement of basal ganglia of the brain is more common when the source of the infection is spreading through hematogenous transmission $(3,7,8)$. Our patient is one of the very rare cases in which the infection was presented in hemispheres of cerebellum without the involvement of the skull. 


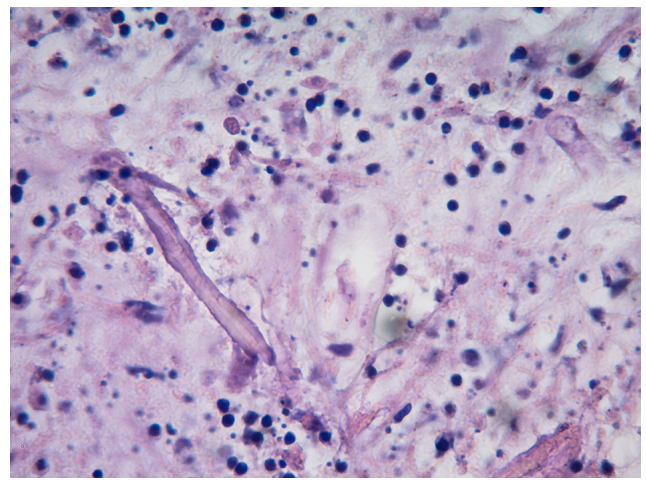

Figure 3. Histopathologic Section of the Specimen, Revealing the Diagnosis of Mucormycosis $(100 \times)$
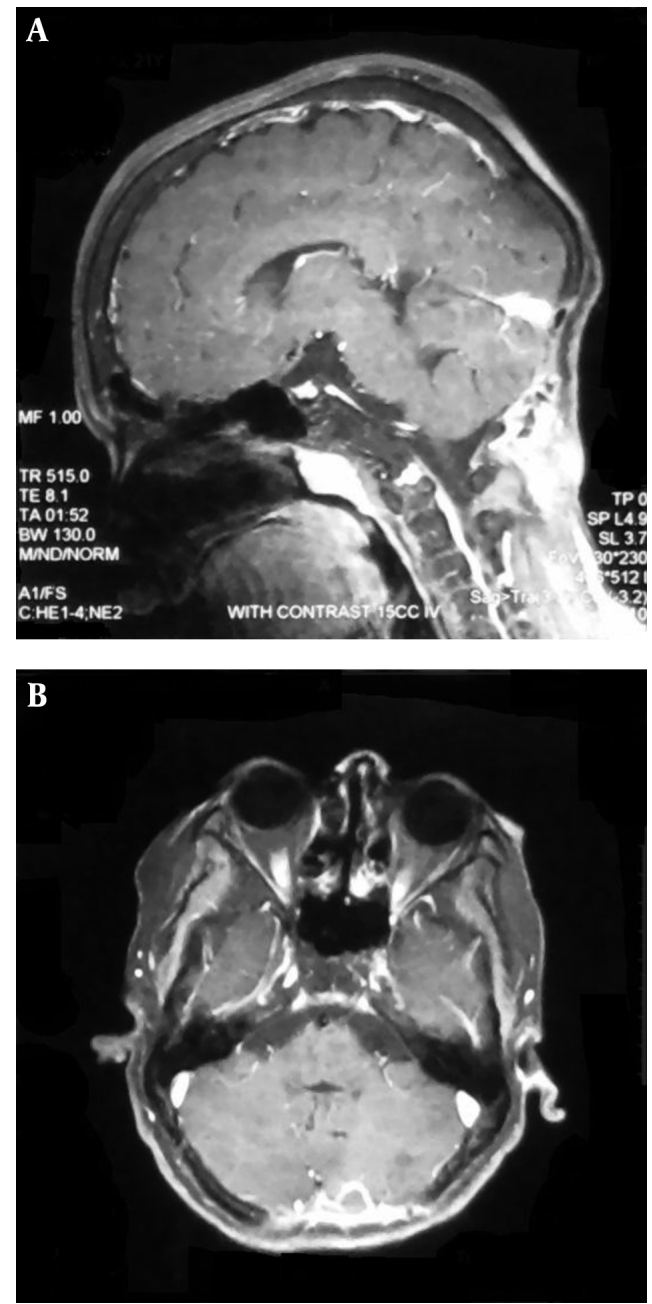

Figure 4. Sagittal (A) and Axial (B) Preoperative MRI of the Patient Showing Resolved Lesion
Diabetes mellitus is the most prevalent predisposing factor in patients with mucormycosis of the brain (9). Our patient was a known case of diabetes mellitus and thalassemia major with a history of frequent blood transfusion and use of deferoxamine as an iron chelator. Several cases of mucormycosis have been reported in which there was a history of deferoxamine administration (10). It is believed that mucorales can absorb more iron through binding to the iron-deferoxamine complex, leading to facilitation of their growth $(11,12)$.

The clinical course of the disease in our patient was slow and after a period of fever and cough, it was presented as vertigo and imbalance. The majority of reports have indicated an acute and progressive course although some chronic cases of pulmonary, paranasal, and intracranial mucormycosis have been reported (1).

Early treatment is the cornerstone of rescuing the patient. Surgical debridement and radical resection in cases of brain abscesses and rhino-sinuso-orbital involvement can save the patient's life. Beside surgical treatment, medical treatment with antifungal drugs is necessary.

The single lesion on the patient's MRI report and slow progression of the disease misled us to the diagnosis of brain tumor. The patient therefore underwent surgery and biopsy of the mass lesion. Histological findings of the sample revealed mucormycosis. Intravenous treatment with $1-1.5 \mathrm{mg} / \mathrm{kg} /$ day of amphotericin deoxycolate was commenced and continued for 3 weeks. Regarding the vitality of host iron availability for pathogenesis of mucormycosis, deferasirox 15-20 mg/day was administered for the patient, which was further discontinued on the eighth day due to increased serum creatinine level. However, the safety of this combination therapy is still controversial and administration of deferasirox is not recommended in mucormycosis patients because a higher mortality with this drug was observed in trials $(13,14)$. The patient was discharged in good general conditions and the control MRI showed no sign of recurrence and confirmed complete resolution.

\section{References}

1. Air EL, Vagal AA, Kendler A, McPherson CM. Isolated cerebellar mucormycosis, slowly progressive over 1 year in an immunocompetent patient. Surg Neurol Int. 2010;1:81. doi: 10.4103/2152-7806.73800. [PubMed: 21206542].

2. Bhadada S, Bhansali A, Reddy KS, Bhat RV, Khandelwal N, Gupta AK. Rhino-orbital-cerebral mucormycosis in type 1 diabetes mellitus. Indian J Pediatr. 2005;72(8):671-4. doi: 10.1007/BF02724075. [PubMed: 16131772].

3. Verma A, Brozman B, Petito CK. Isolated cerebral mucormycosis: report of a case and review of the literature. J Neurol Sci. 2006;240(12):65-9. doi: 10.1016/j.jns.2005.09.010. [PubMed:16269155].

4. Cook BA, White CB, Blaney SM, Bass JW. Survival after isolated cerebral mucormycosis. Am J Pediatr Hematol Oncol. 1989;11(3):330-3. [PubMed: 2782563]. 
5. Eucker J, Sezer O, Lehmann R, Weber JR, Graf B, Denkert C, et al. Disseminated mucormycosis caused by Absidia corymbifera leading to cerebral vasculitis. Infection. 2000;28(4):246-50. doi: 10.1007/s150100070047. [PubMed: 10961535].

6. Grannan BL, Yanamadala V, Venteicher AS, Walcott BP, Barr JC. Use of external ventriculostomy and intrathecal anti-fungal treatment in cerebral mucormycotic abscess. J Clin Neurosci. 2014;21(10):1819-21. doi: 10.1016/j.jocn.2014.01.008. [PubMed: 24852901].

7. Hopkins RJ, Rothman M, Fiore A, Goldblum SE. Cerebral mucormycosis associated with intravenous drug use: three case reports and review. Clin Infect Dis. 1994;19(6):1133-7. doi: 10.1093/clinids/19.6.1133. [PubMed: 7888545].

8. Terk MR, Underwood DJ, Zee CS, Colletti PM. MR imaging in rhinocerebral and intracranial mucormycosis with CT and pathologic correlation. Magn Reson Imaging. 1992;10(1):81-7. doi: 10.1016/0730725X(92)90376-B. [PubMed: 1545686].

9. Lanternier F, Dannaoui E, Morizot G, Elie C, Garcia-Hermoso D, Huerre M, et al. A global analysis of mucormycosis in France: the RetroZygo Study (2005-2007). Clin Infect Dis. 2012;54 Suppl 1:S35-43. doi: 10.1093/cid/cir880. [PubMed: 22247443].
10. Daly AL, Velazquez LA, Bradley SF, Kauffman CA. Mucormycosis: association with deferoxamine therapy. Am J Med. 1989;87(4):468-71. doi 10.1016/S0002-9343(89)80836-8. [PubMed: 2679077].

11. Artis WM, Patrusky E, Rastinejad F, Duncan RJ. Fungistatic mechanism of human transferrin for Rhizopus oryzae and Trichophyton mentagrophytes: alternative to simple iron deprivation. Infect Immun. 1983;41(3):1269-78. [PubMed: 6885162].

12. Holzberg M, Artis WM. Hydroxamate siderophore production by opportunistic and systemic fungal pathogens. Infect Immun. 1983;40(3):1134-9. [PubMed: 6221998].

13. Spellberg B, Ibrahim A, Roilides E, Lewis RE, Lortholary O, Petrikkos G, et al. Combination therapy for mucormycosis: why, what, and how? Clin Infect Dis. 2012;54 Suppl 1:S73-8. doi:10.1093/cid/cir885. [PubMed: 22247449]

14. Spellberg B, Ibrahim AS, Chin-Hong PV, Kontoyiannis DP, Morris MI, Perfect JR, et al. The Deferasirox-AmBisome Therapy for $\mathrm{Mu}$ cormycosis (DEFEAT Mucor) study: a randomized, double-blinded, placebo-controlled trial.JAntimicrob Chemother.2012;67(3):715-22. doi: 10.1093/jac/dkr375. [PubMed: 21937481]. 